\title{
Peak expiratory flow changes during experimental rhinovirus infection
}

\author{
P.G. Bardin***, D.J. Fraenkel*, G. Sanderson*,+, E.M. van Schalkwyk**, \\ S.T. Holgate*, S.L. Johnston*,
}

Peak expiratory flow changes during experimental rhinovirus infection. P.G. Bardin, D.J. Fraenkel, G. Sanderson, E.M. van Schalkwyk, S.T. Holgate, S.L. Johnston. (C) ERS Journals Ltd 2000.

ABSTRACT: Rhinovirus (RV) colds are associated with asthma exacerbations and experimental infections are commonly used to investigate the mechanisms involved. However, a temporal association between experimental RV infections and falls in peak expiratory flow (PEF) have not been demonstrated.

PEF was measured in 22 volunteers (11 normal, five atopic, six atopic asthmatic) who developed RV serotype 16 colds after inoculation. PEF was measured twice daily for 2 weeks prior and 6 weeks after RV infection and episodes of respiratory morbidity based on changes in PEF were defined using validated criteria.

Six significant reductions in PEF were temporally related to the RV infections (in two $(18 \%)$ normal, one $(20 \%)$ atopic, three $(50 \%)$ atopic asthmatic subjects, $p=0.1)$ and occurred 4-9 days (median 6) after inoculation. Mean \pm SEM PEF at day 6 was $87.8 \pm 1.8 \%$ of the predicted value in the six subjects with reductions versus $99.4 \pm 1.4 \%$ pred in those without $(p=0.01)$. Symptom scores were significantly different at day 6 in the two groups $(10.6 \pm 1.9$ versus $6.8 \pm 1.0, p=0.03)$, but no differences were noted in the viral culture scores and changes in nasal albumin. In subjects with significant PEF reduction, the decrease in the provocative concentration causing a $20 \%$ fall in the forced expiratory volume in one second (FEV1) (PC20) was $1.7 \pm 1.3 \mathrm{mg} \cdot \mathrm{mL}^{-1}$ versus $1.2 \pm 1.1 \mathrm{mg} \cdot \mathrm{mL}^{-1}$ in the negative group $(p=0.06)$. The degree of seroconversion to $R V$ was significantly higher in the group with reduced PEF (median change dilutions 8 versus $4, \mathrm{p}=0.02$ ).

The results of the present study suggest that rhinovirus-associated, respiratory morbidity occurs during experimental infection in some but not all normal and asthmatic subjects and also that experimental colds are a valid model for the study of rhinovirus-associated airway symptoms and asthma exacerbations.

Eur Respir J 2000; 16: 980-985.
*University Medicine, Southampton General Hospital, Southampton, UK. **Lung and Allergy Unit and Internal Medicine, University of Stellenbosch, Cape Town, South Africa. ${ }^{+}$National Heart and Lung Institute, Imperial College School of Medicine at St Mary's, London, UK.

\section{Correspondence: P.G. Bardin}

Lung and Allergy Unit/Internal Medicine School of Medicine, University of Stellenbosch,

PO Box 19063, Tygerberg 7505

Cape Town

South Africa.

Fax: 27219333591

Keywords: Asthma experimental infection peak expiratory flow rhinovirus

Received: December 281999

Accepted after revision August 22000
Common cold viruses, particularly rhinovirus (RV) have been implicated as a major trigger for asthma exacerbations. Using a sensitive polymerase chain reaction (PCR) assay, JoHnston et al. [1] found a significant association between episodes of respiratory morbidity and viral identifications, the majority of viruses being RV. In adults $44 \%$ of similar decreases in peak expiratory flow (PEF) were found to be associated with viral infection, again the majority were RV [2]. In both studies a clear temporal relationship between reductions in PEF and the presence of viruses was demonstrated, suggesting physiologically relevant effects of viral infections on lower airway function. A recent study by REDDEL et al. [3] demonstrated a distinctive pattern of decline in PEF during asthma exacerbations that was different to that found during undertreated asthma with poor control. Although viral identification was not carried out, more than $90 \%$ of exacerbations were temporally associated with symptoms consistent with a diagnosis of upper respiratory infections. Modulation of lower airway function by upper respiratory viral infection is, therefore, of considerable interest and importance and elucidation of the relevant mechanisms may indicate new avenues for treatment of virus-induced asthma.

Experimental RV infections in volunteers have provided a valuable model to study pathophysiological mechanisms linking respiratory viral infections to lower airway symptoms [4]. Using this method, recent experimental studies have demonstrated new late asthmatic reactions to allergen provocation in association with infection [5], similar new responses associated with increased allergen induced eosinophilia [6], T-cell recruitment to the bronchial submucosa [7] and increases in interleukin 8 (IL-8) and eosinophil cationic protein [8]. Similar studies also detected prolonged increases in airway responsiveness [9], and portable spirometry measuring forced expiratory volume in one second $(\mathrm{FEV} 1)$ has been shown to decrease by approximately $10 \%$ in atopic asthmatics [10]. However, no previous experimental studies have demonstrated changes in PEF measurements similar to the distinctive pattern of PEF reduction observed in community studies $[1,2]$. The relevance of experimental $\mathrm{RV}$ disease to 
asthma exacerbations is therefore, still in some doubt as well as the immunological, clinical and other implications suggested by the results of various studies based on this model.

Serial PEF measurements in normal and asthmatic subjects before, during and after experimental RV infection were examined in this study. Because it may be difficult to define an asthma exacerbation consistently on the basis of PEF only, a definition of an episode of respiratory morbidity was employed that was developed and validated in longitudinal studies [11]. This definition is also similar to that employed to detect exacerbations of asthma in a recent Australian study [3]. Using these criteria applied to daily PEF measurements, significant episodes of airway obstruction could be identified in a subgroup of subjects who also experienced more severe colds and greater increases in airway responsiveness. The results suggest that experimental RV infection is an appropriate and relevant model for clinical RV disease and asthma exacerbations.

\section{Materials and methods}

\section{Patients and study design}

The study was conducted in 22 (11 normal, five atopic nonasthmatic and 6 atopic asthmatics) nonsmoking volunteers, who had low or absent titres of neutralizing antibody to RV16 in their serum. Asthmatic subjects were required to use only "as needed" beta $(\beta)$-agonist inhalation treatment, to be atopic with $\geq 1$ positive skin test reaction to allergen and to not have used any nasal, oral or inhaled corticosteroids within the prior 6 weeks. The studies were approved by the Southampton and District Ethics Committee.

Subjects were initially screened for neutralizing antibody and then studied in three phases. Phase 1: baseline observations were conducted 2 days prior to viral inoculation and included serum for repeat neutralizing antibody testing and nasal washing for viral culture; phase 2: took place in the Infectious Diseases Unit (IDU) at Southampton General Hospital, where subjects were isolated for 7 days during the experimental RV16 infection. Following RV16 inoculation, subjects had daily nasal washes, assessment of symptoms and repeat serology on day 5; phase 3: all investigations were repeated at weeks 6-8.

\section{Allergen skin prick testing}

Testing was performed as previously described [12] using allergen extracts. Histamine $\left(1.8 \mathrm{mg} \cdot \mathrm{mL}^{-1}\right)$ was the positive control employed for the investigations. Skin reactivity was assessed after $15 \mathrm{~min}$ and considered positive if $a \geq 3 \mathrm{~mm}$ wheal surrounded by erythema developed.

\section{Nasal inoculation with RV16}

Nasal inoculation was performed using $200050 \%$ tissue culture infection dose (TCID50) of RV16 as described previously [12]. RV16 (a kind gift from W. Busse and E.
Dick, Madison, Wisconsin), was used to inoculate the first 17 volunteers who participated in the studies. Thereafter, a new RV16 inoculum was prepared and used to induce colds in the subsequent five subjects [4].

\section{Symptom assessment}

All subjects completed a validated questionnaire daily for 2 weeks prior to, during and for 6-8 weeks after RV 16 infection. Cold symptoms were graded $0-3$ according to severity, and the criteria for a cold were based on those proposed by JACKSON et al. [13]. Clinical illness was considered to be present if a volunteer had a minimum cumulative symptom score of 14 over a 4 day period, and had a subjective impression that they had a cold, or if rhinorrhoea was present on at least 3 of the 5 days of observation. Median baseline rhinitic symptoms present for 4 days prior to inoculation were subtracted from new cold symptoms.

\section{Nasal lavage and viral culture}

Nasal washing was undertaken by instilling $4 \mathrm{~mL}$ of prewarmed Hank's balanced salt solution (HBSS) with $0.5 \%$ gelatin into each nostril, holding for $5 \mathrm{~s}$ and then expelling into a sterile Petri dish. Samples were divided into aliquots and frozen at $-70^{\circ} \mathrm{C}$ until analysed.

Nasal washes were inoculated into Ohio Hela cell cultures, and RV16 infection was diagnosed by means of the typical cytopathic effects (CPE) on light microscopy and confirmed by neutralization using RV16 specific antiserum (ATCC. Cat. No.V105-501-558; Bethesda, MD, USA). Cultures were examined daily and regarded as negative if they showed no CPE after two passes in cell culture. Positive infection was established by at least one identified viral isolate and/or a four-fold rise in titre of neutralizing antibody. A scoring procedure for viral culture was employed to reflect the amount of virus shed [4]. Daily nasal washes were cultured and given CPE scores (range 0-4); the scores were then combined for the 3 day period of lavage collection, with a maximum cumulative score of $12(3 \times 4)$.

\section{Measurement of neutralizing antibodies}

The measurement of neutralizing antibodies was undertaken on serum from all volunteers before admission to the study (screening) as well as on the day prior to inoculation with RV16, on Day 5 of experimental infection and finally, 6-8 weeks later, as described previously [12]. Results are expressed as fold difference from baseline.

\section{Albumin measurement}

Measurements on coded samples (baseline and day 5) were performed by rocket immunoelectrophoresis. Sensitivity of the assay was $1 \mu \mathrm{g} \cdot \mathrm{mL}^{-1}$ and the coefficient of variation of repeated measurements was $<5 \%$ [14]. 


\section{PEF measurements}

Measurements were performed (morning and evening) by all subjects for 2 weeks prior to and 6 weeks after infection, using a mini-Wright peak flow meter (Clement Clarke International, London, UK). At enrolment all the subjects received thorough instructions in the use of the instrument and their technique was rechecked during the period of their hospital admission. The best of 3 measurements was noted and the highest value recorded on a diary card. For analysis all PEF measurements were expressed as \% predicted (\% pred). Analysis of PEF data as amplitude $\%$ mean or $\%$ maximum was also carried out and yielded similar results

\section{Episodes of respiratory morbidity}

Episodes of respiratory morbidity were identified and defined as a fall in morning PEF $>1.5$ standard deviations (SD) below that subjects' mean morning PEF for at least 2 consecutive days, PEF being $>1.5$ SD below the mean for 1 day before, and 2 consecutive days after the episode [11]. A diagrammatic representation is shown in figure 1. Data from all subjects were plotted graphically, to facilitate the identification of patterns of disease and associations between events by visual inspection.

\section{Measurements of airway responsiveness}

Histamine bronchoprovocation was undertaken at baseline ( 2 days prior to infection), and on day 5 after infection using a standard bronchial provocation technique, adapted from the method of CHAI et al. [15]. Doubling concentrations of histamine $\left(0.03-32.0 \mathrm{mg} \cdot \mathrm{mL}^{-1}\right)$ were administered via an Inspiron nebulizer until FEV1 fell by $>20 \%$ of post-saline values. The provocative concentration of histamine producing a $20 \%$ fall in FEV1 (PC20) was derived by linear interpolation of the log-concentration response curve. Subjects in whom a PC20 could not be measured using the highest concentration, was calculated by extrapolation for one doubling dilution beyond the highest concentration administered [16].

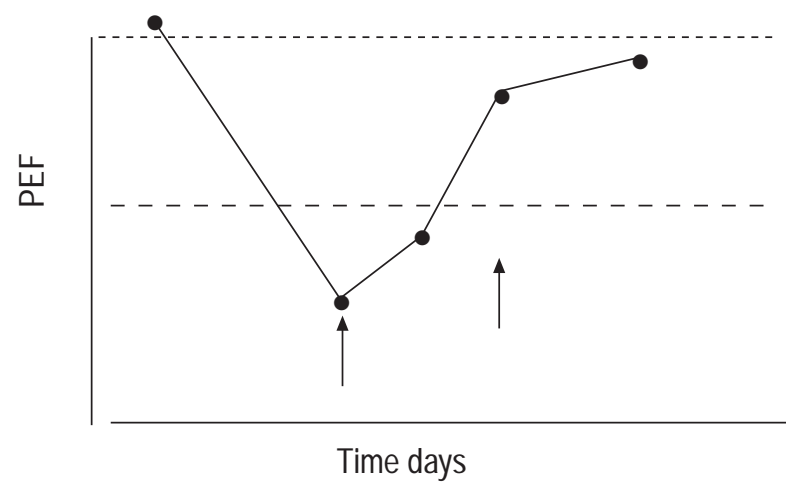

Fig. 1. - Diagrammatic representation of an episode of respiratory morbidity. A fall in PEF $>1.5$ SD below the mean for at least 2 days is followed by PEF $>1.5$ SD for at least 2 consecutive days. PEF: peak expiratory flow. (- - ): 0 ; mean; -- : $1.5 \mathrm{SD}$; arrows represent start and end points.

\section{Statistical analysis}

All data was transferred to a computer spreadsheet and checked against the original source data. Summary data for symptom scores, PEF measurements, PC20 measurements and other relevant variables were obtained. Data were expressed as mean \pm SEM except for albumin, seroconversion and culture scores which were expressed as median values.

The number of PEF episodes occurring, during the 14 days after experimental inoculation was determined as well as those starting during the 2 weeks prior to inoculation and the final 4 weeks of convalescence. The cumulative duration of episodes (days PEF was $<1.5 \mathrm{SD}$ ) was calculated as well as the magnitude (average number of SD below the mean to which PEF fell during episodes). Episodes of respiratory morbidity were compared between the different times using $\mathrm{X}^{2}$-analysis. Other variables including symptom scores, nasal albumin measurements and changes in $\mathrm{PC} 20$ (log transformed) were assessed using the Mann-Whitney U-test, Kruskal Wallis or paired t-test as appropriate. A p-value of $<0.05$ was regarded as significant.

\section{Results}

\section{Patients}

The 22 subjects completed the 8 weeks of study successfully and none of the 6 asthmatics had an exacerbation requiring hospital admission or changes in treatment. Infection was confirmed in all 22 subjects by RV16positive culture of nasal lavage and/or a $\geq$ four-fold rise in neutralizing antibody titres. The subjects demographic and other baseline data are shown in table 1 .

\section{Episodes of respiratory morbidity}

Over the 8 weeks of study, a total of six PEF episodes occurred in the 14 day period following inoculation in two normal (18\%) subjects, one (20\%) atopic and three (50\%) atopic asthmatic subjects ( $\mathrm{p}$-value for trend=0.1). All started 4-9 days post-inoculation (median 6) and were clearly temporally related to the period of the RV16 experimental cold and cold symptoms. On day 6 PEF measurements (\% pred) were significantly lower $(87.8$ $\pm 1.8 \%$ ) in those subjects with an episode of respiratory morbidity compared to those subjects without an episode $(99.4 \pm 1.4 \% ; \mathrm{p}=0.03)$. A typical example is shown in figure 2 , an asthmatic subject who had stable PEF measurements until 6 days after inoculation with RV16 (fig. 2). Significant decreases were noted for 2 subsequent days with moderate lower respiratory symptoms (cough), followed by recovery and stable PEF measurements over the next 5 weeks. Similar patterns were observed in the other five subjects including two normal individuals (patients 6 and 7 , table 1) with no evidence of atopy or history of asthma. Interestingly, both subjects had a severe cold as measured by cold scores on day 6 (scores 18 and 10), notable changes in $\mathrm{PC} 20\left(>32 \mathrm{mg} \cdot \mathrm{mL}^{-1}\right.$ before, 5.7 and 12.3 $\mathrm{mg} \cdot \mathrm{mL}^{-1}$ respectively after, $\left.\mathrm{RV} 16\right)$ and seroconversion with marked increases in neutralizing antibody (X8). 
Table 1. - Baseline characteristics of 22 subjects with experimental RV16 infection

\begin{tabular}{|c|c|c|c|c|c|c|c|c|}
\hline Subject & Age/Sex & Group & $\begin{array}{l}\text { PEF } \\
\% \text { pred }\end{array}$ & $\begin{array}{c}\mathrm{PC} 20 \\
\mathrm{mg} \cdot \mathrm{mL}^{-1}\end{array}$ & $\begin{array}{c}\text { Cold score } \\
\text { D6 }\end{array}$ & $\begin{array}{l}\text { Culture } \\
\text { score* }\end{array}$ & $\begin{array}{c}\text { Serocon } \\
\text { version** }\end{array}$ & Treatment \\
\hline 1 & $50 / \mathrm{M}$ & $\mathrm{N}$ & 105 & 21.5 & 2 & 12 & 16 & - \\
\hline 2 & $45 / F$ & $\mathrm{~N}$ & 100 & 32 & & 12 & 2 & - \\
\hline 3 & $45 / \mathrm{F}$ & $\mathrm{N}$ & 101 & $>32$ & 9 & 12 & ND & - \\
\hline 4 & $20 / \mathrm{F}$ & $\mathrm{N}$ & 100 & $>32$ & 4 & 4 & 0 & - \\
\hline 5 & $21 / \mathrm{M}$ & $\mathrm{N}$ & 105 & $>32$ & 3 & 12 & 8 & - \\
\hline 6 & $20 / F$ & $\mathrm{~N}$ & 98 & $>32$ & 10 & 12 & 8 & - \\
\hline 7 & $29 / \mathrm{M}$ & $\mathrm{N}$ & 95 & $>32$ & 18 & 12 & 8 & - \\
\hline 8 & $49 / \mathrm{M}$ & $\mathrm{N}$ & 103 & 4.0 & 6 & 12 & 2 & - \\
\hline 9 & $24 / \mathrm{F}$ & $\mathrm{N}$ & 107 & 9.2 & 4 & 10 & ND & - \\
\hline 10 & $43 / \mathrm{F}$ & $\mathrm{N}$ & 79 & $>32$ & 4 & 10 & 64 & - \\
\hline 11 & $26 / F$ & $\mathrm{~N}$ & 102 & 17.9 & 10 & 10 & 32 & - \\
\hline 12 & $47 / F$ & A & 98 & 25.4 & 1 & 8 & 2 & - \\
\hline 13 & $33 / \mathrm{M}$ & A & 100 & 4.2 & 11 & 4 & 0 & - \\
\hline 14 & $32 / \mathrm{M}$ & A & 101 & $>32$ & 5 & 8 & 32 & - \\
\hline 15 & $27 / M$ & A & 97 & $>32$ & 18 & 12 & 4 & - \\
\hline 16 & $26 / \mathrm{M}$ & A & 99 & $>32$ & 14 & 6 & 32 & - \\
\hline 17 & $20 / \mathrm{M}$ & AA & 97 & 1.1 & 3 & 2 & 0 & $\mathrm{~B}_{2}$ \\
\hline 18 & $22 / \mathrm{M}$ & AA & 87 & 0.6 & 5 & 5 & 32 & $\mathrm{~B}_{2}$ \\
\hline 19 & $23 / \mathrm{M}$ & AA & 100 & 22.0 & 6 & 12 & 4 & $\mathrm{~B}_{2}$ \\
\hline 20 & $26 / F$ & AA & 96 & 0.3 & 8 & 12 & 16 & B? \\
\hline 21 & $22 / \mathrm{M}$ & AA & 96 & 1.6 & 7 & 11 & 64 & $\mathrm{~B}_{2}$ \\
\hline 22 & $22 / \mathrm{M}$ & AA & 96 & 4.0 & 7 & 11 & 64 & $\mathrm{~B}_{2}$ \\
\hline
\end{tabular}

PEF: peak expiratory flow; $\mathrm{PC} 20$ : provocative concentration causing a $20 \%$ fall in forced expiratory volume in one second; N: normal; A: atopic; AA: atopic asthmatic; $\beta_{2}$ : $\beta_{2}$-agonists. PEF and PC20 are baseline measurements. *: see methods for details; **: expressed as fold difference from baseline. Patients $6,7,16,19,21$ and 22 had significant episodes of respiratory morbidity in the first week after inoculation with RV16.

Two further episodes of respiratory morbidity were noted in two asthmatic subjects (one also had an episode after experimental RV16 infection) that appeared unrelated to RV experimental infection. Both occurred 4-6 weeks after inoculation and were not associated with significant wheezing requiring intervention or other signs of an asthma exacerbation. Both subjects did however, experience increases in both upper and lower airway symptoms as judged by diary cards completed during this period suggesting that these episodes may have been related to wild type colds. No episodes of respiratory morbidity were identified in the 2 weeks prior to RV16 inoculation.

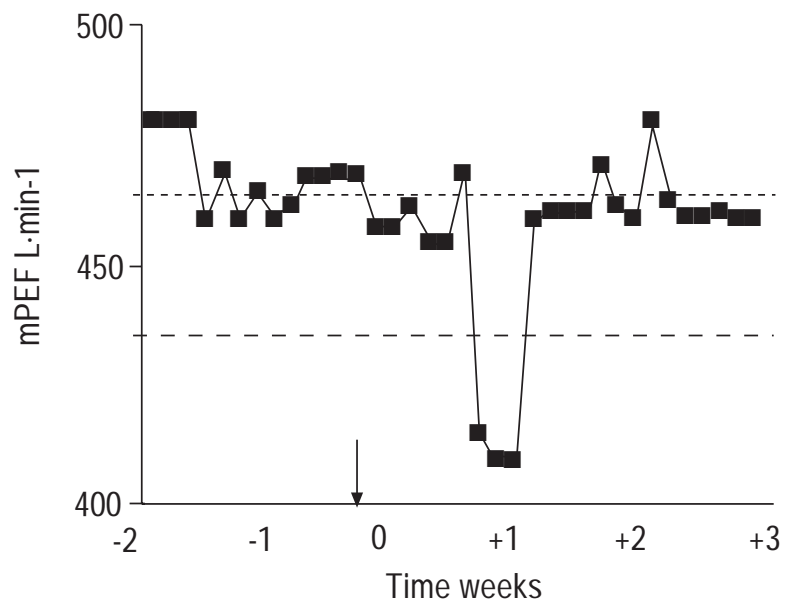

Fig. 2. - Typical pattern of reduction in PEF measurements observed in six subjects associated with experimental RV16 infection and the two subjects with putative wild-type infections. Example is from an asthmatic patient who experienced moderate cold symptoms, cough and mild wheezing. mPEF: morning peak expiratory flow. (- - -): mean; - - : $1.5 \mathrm{SD}$. Arrow represents inoculation point.
Comparing the episodes of morbidity occurring in the 14 days after RV inoculation (related to experimental infection) to those occurring during the rest of the study period, (putatively related to wild type infections), the median duration of significant morbidity was 2 days and 2 and 3 days, respectively. The magnitude of PEF reductions was also similar in the 2 periods.

\section{Effect of other variables}

To assess the possible role of other measured variables, subjects with post-inoculation episodes of respiratory morbidity were compared to those individuals without changes (table 2). Cold scores (mean $\pm(\mathrm{SD}))$ were significantly

Table 2. - Association of episodes of respiratory morbidity with other variables

\begin{tabular}{|c|c|c|c|}
\hline \multirow{2}{*}{ Variable } & \multicolumn{2}{|c|}{ Episode } & \multirow{2}{*}{ p-value } \\
\hline & Present & Absent & \\
\hline Subjects $n$ & 6 & 16 & \\
\hline Total cold score* & $24 \pm 2.1$ & $18 \pm 1.8$ & 0.1 \\
\hline Cold score*, day 6 & $10.6 \pm 1.9$ & $6.8 \pm 1.0$ & 0.03 \\
\hline Cough & 4 & 4 & 0.09 \\
\hline Wheeze & 2 & 2 & 0.3 \\
\hline $\begin{array}{l}\text { Nasal albumin } \mathrm{mg} \cdot \mathrm{mL}^{-1} \\
\text { (change D6-DO) }\end{array}$ & 25 & 15 & 0.3 \\
\hline Degree seroconversion $^{+}$ & $\mathrm{X} 8$ & $\mathrm{X} 4$ & 0.02 \\
\hline PC20 change* $\mathrm{mg} \cdot \mathrm{mL}^{-1}$ & $1.7 \pm 1.3$ & $1.2 \pm 1.1$ & 0.06 \\
\hline Viral culture scores ${ }^{+}$ & 12 & 10 & 0.4 \\
\hline
\end{tabular}

Data are presented as: $*$ : mean $\pm \mathrm{SD} ;{ }^{+}$: median values. $\mathrm{PC} 20$ : provocative concentration causing a $20 \%$ fall in forced expiratory volume in one second. 
different at day 6 in the 2 groups $(10.6 \pm 1.9$ versus $6.8 \pm 1.0, \mathrm{p}=0.03$ ), as was the degree of seroconversion to $\mathrm{RV}$ (median fold increase dilutions 8 versus $4, \mathrm{p}=0.02$ ). However, no differences in total symptom scores were found ( $24 \pm 2.1$ versus $18 \pm 1.8, \mathrm{p}=0.1)$. No differences between groups were noted with regard to lower airway symptoms (cough, wheeze), viral culture scores or changes in nasal albumin. Increases in airway hyperresponsiveness in subjects with respiratory morbidity measured as reductions in $\mathrm{PC}_{20}$ was $1.7 \pm 1.3 \mathrm{mg} \cdot \mathrm{mL}^{-1}$ compared to $1.2 \pm 1.1 \mathrm{mg} \cdot \mathrm{mL}^{-1}$ in the other group, a difference that approached significance $(p=0.06)$. The increases in responsiveness represented mean changes of $0.3 \pm 0.4$ doubling doses in the group with respiratory morbidity and $0.2 \pm 0.4$ in those without such episodes.

\section{Discussion}

The validity of experimental RV disease to serve as a model of episodes of respiratory morbidity in normal and asthmatic subjects has been examined. Significant decreases in PEF were noted which were temporally related to RV experimental colds and occurred 4-9 days after inoculation. Episodes of respiratory morbidity were associated with higher cold scores and a greater degree of seroconversion, both measurements indicative of more severe colds. Patients with PEF changes also tended to have greater associated increases in airway responsiveness during RV infections.

The application of molecular biological techniques and the development of sensitive and reliable polymerase chain reaction assays (PCR) for picornaviruses (PV) and other viruses [1] have made it possible to clarify the important role of viruses in exacerbations of asthma. Longitudinal studies by Johnston et al. [1] and Nicholson et al. [2] have demonstrated an association between PV (predominantly RV) and decreases in PEF with increases in asthma symptoms in $40-80 \%$ of adults and children. These observations in turn have motivated more mechanistic studies in volunteers using experimental RV disease after direct inoculation of the upper airways [4]. However, the relevance of these experimental findings and their possible application to the wild type infections occurring in the longitudinal community studies and their results have remained uncertain. One possible way to link both groups of studies would be to demonstrate asthma "exacerbations" during human experimental disease that are similar to those found in community studies. To date this is limited to evidence that RV experimental infections induce increased bronchial hyperresponsiveness [9] and small changes in FEV1 [10]. However, the majority of community studies on asthma exacerbations have used changes in PEF to define the exacerbations. To date no evidence that alterations in PEF occur during experimental infections has been provided, GRÜNBERG et al. [10] specifically failed to observe any such changes. This study has demonstrated the association for the first time, and helps to validate experimental infection as a valid model for wild type infections. However, it needs to be remembered that these are not observations of "real life" but a model of them, given that it would be ethically unjustified to induce "real" exacerbations.
The inability of previous workers to demonstrate clear episodes of asthma worsening is probably related to the selection of patients with mild asthma for experimental RV studies. Such volunteers are unlikely to develop acute severe airway obstruction and changes in airflow measurements during and after RV infection may therefore, be subtle and transient. One method to examine and identify mild exacerbations is to measure PEF daily and to predefine an "exacerbation" using validated criteria for episodes of respiratory morbidity as employed by CLOUGH and Holgate [11]. The authors investigated PEF data generated as part of the experimental RV program, using this method. The results demonstrate eight episodes of morbidity in seven subjects, six episodes occurring in the week following RV16 inoculation. Three of the six asthmatics studied as well as three nonasthmatic individuals, had episodes within 14 days indicating that in susceptible asthmatic airways as well as normal subjects, virusassociated reductions in airflow can be identified. The temporal association of these episodes to experimental $\mathrm{RV}$ disease implies that they may be linked and it appears likely that similar reductions in airflow may occur both in experimental and naturally occurring RV infections.

Peak-flow charts demonstrated a distinctive pattern (fig. 2) during episodes of respiratory morbidity. A linear decline in consecutive PEF values was followed by a sharp point of inflection, again followed by a linear increase in PEF measurements. The pattern is similar to that observed by ReDDEL et al. [3] who demonstrated differences in PEF patterns after asthma exacerbations (presumed viral) compared to that observed in poorly controlled disease. The pattern is also similar to that seen during RV-associated decreases in PEF in earlier community studies [1]. It is possible that the small number of PEF measurements (twice daily) or the inaccuracy of the mini-Wright PEF meter in the mid flow range may have had some confounding effects. However, this is unlikely given the large changes observed and the strict criteria used to define episodes of morbidity.

Two episodes of morbidity were noted in two asthmatic subjects during the convalescent period (one also had an episode related to RV16). Because studies were not designed to examine the presence of viral infections or other triggers in this period, it is not known whether these changes in PEF may be related to RV or other factors.

Interestingly, subjects with episodes of morbidity tended to have greater increases in airway responsiveness, one mechanism linked to the worsening of asthma. Studies by the authors group [7] and others [5, 9] have demonstrated increases in airway reactivity that occur during experimental RV infections. The data in this study extend these observations to show that increased reactivity during RV infection may be associated with greater changes in airflow limitation, and hence, with unstable asthma.

It is of interest to know whether there may be predictors of a worsening of airway function. Severity of an RV cold could be the primary determinant as reflected by severity of symptoms, degree of seroconversion and levels of nasal albumin [17]. It has been previously demonstrated that atopic individuals develop more severe colds [12], and in this group of seven subjects with changes in PEF, five were atopic on skin testing. Other factors associated with episodes of morbidity included high symptom scores (day 6 after cold) and degree of seroconversion, both 
measures likely to reflect severity of infection. Nasal levels of albumin were not significantly different in the 2 groups, probably because the number of subjects who had episodes of morbidity was too small for valid comparisons. However, it remains possible that the severity of the RV cold was related to the degree of nasal and also endobronchial inflammation produced and that this was reflected as measureable reductions in airway function. Future biopsy studies of the nose and lower airway should clarify whether altered inflammatory cells and mediators result in the observed changes.

The results validate experimental rhinovirus infection as a model for natural rhinovirus disease and its consequences and effects in asthmatics. Experimental studies with endobronchial biopsy have provided considerable insight into the inflammatory changes accompanying lower airway rhinovirus infection $[7,18,19]$. Further experimental investigations will help to define the mechanisms whereby lower airway function is compromised and clarify how asthma is exacerbated by viruses.

Acknowledgements. The authors would like to thank all the volunteers who participated in these studies. Dedicated patient care was given by $\mathrm{M}$. Dorward and G. Smith and expert secretarial assistance was given by S. Stipp. Measurements of nasal albumin were performed by L.C.K. Lau.

\section{References}

1. Johnston SL, Pattemore PK, Sanderson G, et al. Community study of role of viral infections in exacerbations of asthma in 9-11 year old children. BMJ 1995; 310: 12251229.

2. Nicholson KG, Kent J, Ireland DC. Respiratory viruses and exacerbations of asthma in adults. BMJ 1993; 307: 982-986.

3. Reddel H, Ware S, Marks G, Salome C, Jenkins C, Woolcock A. Differences between asthma exacerbations and poor asthma control. Lancet 1999; 353: 364-369.

4. Bardin PG, Sanderson G, Robinson BS, Holgate ST, Tyrrell DAJ. Experimental rhinovirus infection in volunteers. Eur Respir J 1996; 9: 2250-2255.

5. Lemanske RF Jr, Dick EC, Swenson CA, Vrtis RF, Busse WW. Rhinovirus upper respiratory infection increases airway hyperreactivity and late asthmatic reactions. J Clin Invest 1989; 83: 1-10.
6. Calhoun WJ, Dick EC, Schwartz LB Busse WW. A common cold virus, rhinovirus 16 , potentiates airway inflammation after segmental antigen bronchoprovocation in allergic subjects. J Clin Invest 1994; 94: 2200-2208.

7. Fraenkel DJ, Bardin PG, Sanderson G, Lampe F, Johnston SL, Holgate ST. Lower airway inflammation during rhinovirus colds in normal and in asthmatic subjects. $\mathrm{Am} \mathrm{J}$ Respir Crit Care Med 1995; 151: 879-886.

8. Grünberg K, Smits HH, Timmers MC, et al. Experimental rhinovirus 16 infection effects cell differentials and soluble markers in sputum in asthmatic subjects. Am J Respir Crit Care Med 1997; 156: 609-616.

9. Cheung D, Dick EC, Timmers MC, et al. Rhinovirus inhalation causes long-lasting excessive airway narrowing in response to methacholine in asthmatic subjects in vivo. Am J Respir Crit Care Med 1995; 152: 1490-1496.

10. Grünberg K, Timmers MC, de Klerk EPA, et al. Experimental rhinovirus 16 (RV16) infection decreases homerecordings of FEV1 in asthmatics. Eur Respir J 1997; 10: (Suppl. 25), 9s.

11. Clough JB, Holgate ST. Episodes of respiratory morbidity in children with cough and wheeze. Am J Respir Crit Care Med 1994; 150: 48-53.

12. Bardin PG, Fraenkel DJ, Sanderson G, et al. Amplified rhinovirus colds in atopic subjects. Clin Exp Allergy 1994; 24: 457-464.

13. Jackson GG, Dowling HIF, Spiesman IG, Goand AV. Transmission of the common cold to volunteers under controlled conditions. Arch Int Med 1958; 101: 267-278.

14. Weeke B. Rocket immunoelectrophoresis. Scand J Immunol 1973; 1: 37-46.

15. Chai H, Froehlich LA, Mathison DA, et al. Standardization of bronchial inhalation challenge procedures. $J$ Allergy Clin Immunol 1975; 56: 322-334.

16. Townley RJ, Hopp RJ. Inhalation methods for the study of airway responsiveness. J Allergy Clin Immunol 1987; 90: 111-124.

17. Naclerio RM, Lichtenstein LM, Kagey-Sobotka A, et al. Kinins are generated during experimental rhinovirus colds. J Infect Dis 1988; 157: 133-142.

18. Sharon EF, Grunberg K, van Krieken JHJM, Sterk PJ. Rhinovirus (RV16) infection enhances ICAM-1 expression in bronchial mucosabiopsies of mildly asthmatic subjects, regardless of inhaled steroid treatment. $\mathrm{Am} \mathrm{J}$ Respir Crit Care Med 1998; 157: A22.

19. Trigg CJ, Nicholson KG, Wang JH, et al. Bronchial inflammation and the common cold a comparison of atopic and non-atopic individuals. Clin Exp Allergy 1996; 26: 665-676. 